ISSN 1112-9867

\title{
MULTISENSORY TRAINING FOR POSTURAL SWAY CONTROL IN NON-INJURED ELDERLY FEMALES
}

\author{
A. C. Lee ${ }^{1, *}$ and D. J. Magee ${ }^{2}$ \\ ${ }^{1}$ Faculty of Sports Science and Coaching, Universiti Pendidikan Sultan Idris, Tg Malim, \\ Perak, Malaysia \\ ${ }^{2}$ Faculty of Rehabilitation Medicine, University of Alberta, Edmonton, Canada
}

Published online: 10 November 2017

\begin{abstract}
Elderly adults demonstrate increased postural sway, which may ultimately lead to falls. The present study used a randomized controlled intervention to investigate whether the three-week multisensory training program lead to a decreased postural sway. A total of 24 non-injured elderly females (60-80 years) volunteered to participate. They were randomly assigned to either a training group (ETG) who received one hour of training twice weekly for three weeks or to a control group (ECG) who received no training. At post-training, the results showed significant improvement in the trained ETG when compared to the untrained ECG for all three postural sway measures for all six training factors. The findings demonstrated that the trained ETG improved in their total Berg Balance Test (BBT) scores indicated that the training program successfully improved postural sway control for non-injured elderly females. Keywords: multisensory training, postural sway control, balance ability, visual input; vestibular input, somatosensory input
\end{abstract}

Author Correspondence, e-mail: aichoo8@hotmail.com

doi: http://dx.doi.org/10.4314/jfas.v9i6s.82 


\section{INTRODUCTION}

Although postural control is taken for granted, it is a complex process involving the coordinated actions of biomechanical, sensory, motor, and central nervous system components [1]. Postural control has been defined as the ability to maintain postural equilibrium in a gravitational field by keeping or returning the center of body mass over its base of support to attain the desired positions or movement without falling [2]. The ability to maintain postural control under dynamic conditions is an important underlying component of physical activity or performance [3]. Deviation from this center of balance in any direction represents postural sway. Postural sway is the distance expressed in centimetres that an individual travels away from his or her center of balance [2].

Stabilization of postural equilibrium is achieved by continuous afferent and efferent control strategies within the sensorimotor system with feedback from somatosensory, vestibular and visual inputs [4]. The afferent information is processed in the brainstem and cerebellum, and then motor commands are initiated [5]. When any of the sensorimotor feedback loops is suppressed or defective, body sway increases and muscle activity increases concurrently, to maintain balance [6].

Proprioceptive input is the cumulative neural input from the mechanoreceptors in the muscles, joint capsules, ligaments, tendons, and skin that is conveyed to the central nervous system (CNS) through afferent neural pathways [7]. Maintaining balance and postural stability are functions of a number of sensory inputs to the CNS, including visual, vestibular, and somatosensory components. These three sensory inputs are required because no single combination of the three senses that provides accurate center of gravity (COG) information under all performance conditions. This is because one or more of the senses may provide information that is misleading or inaccurate for purposes of balance and postural control [1]. During sensory conflict situations, the CNS must quickly select the sensory inputs providing accurate orientation information and must ignore the other misleading ones. Failure to ignore conflicting sensory inputs can lead to instability or surface and surround motion illusions [1]. Despite the availability of multiple sensory inputs, the CNS generally relies on only one sense at a time for orientation information [8].

Aging has impacts virtually on all aspects of the individual sensory and motor components of 
the balance system [9]. Dysfunction in the sensory and motor systems contributes to an increased risk of falling among people 65 or more years of age [10]. Adequate postural control requires intact sensory and motor systems to enable detection of COG deviations and to generate appropriate and prompt muscle responses to effect postural corrections [11]. The elderly who have ineffective sensory processing are unable to adjust and do not demonstrate anticipatory postural reactions in cooperate with the degree of perturbation. The deficits in proprioceptive and kinesthetic processing may be ineffective in altering postural disturbance, hence leading to increased episodes of falling in the elderly [12]. Experimental and clinical evidence suggests a decline in the ability to integrate the three sensory inputs for maintenance of posture is seen in elderly [13].

The aging effects on the musculoskeletal system such as loss of muscle mass, strength, contractile speed, and power has been attributed to changes and the decreased use of the neuromuscular system [14]. These declines will decrease the efficacy of CNS integration to select and adjust muscles contractile patterns, thus slowing the process of sending the decision to peripheral motor components such as muscles acting on ankle, thigh, trunk, and neck for generation of body movement to maintain upright posture and balance control. The slow reaction and response of the peripheral motor component could at the aged at risk to falling [15]. The impact of aging on the deficits of CNS is to alter balance, and impair hearing and sight. Impaired hearing and sight will decrease sensory inputs from vision, and the vestibular region to interact with environmental disturbance to determine the body position to execute the best choice of body movement, thus increasing the risk of fall or imbalance [9]. Sheldon [16] first suggested that the inability to control postural sway in advancing years plays an important part in the tendency of old people to fall. Overstall et al. [17] and Fernie et al. [Erreur ! Source du renvoi introuvable.] related postural sway to falls and suggesting that the amount of the sway amplitudes of fallers were more than non-fallers, when the cause of the fall is other than a trip or slip.

Interestingly, it has been demonstrated that aged subjects are able to decrease the amplitude of postural sway with practice and training [19]. Altered postural responses in elderly subjects, such as delayed onset latencies, intermittent reversal of muscle activation sequences and occasional co-contractions in lower leg muscles, have shown a tendency to improve with 
practice [20]. Hu and Woollacott [Erreur! Source du renvoi introuvable.] found that multisensory balance training designed to improve intersensory interaction could effectively improve balance performance in healthy older adults after receiving a ten-hour balance training program. In addition, Kammerlind et al. [Erreur ! Source du renvoi introuvable.] found that an eight-week balance training in elderly people with non-peripheral vertigo and unsteadiness seemed to improve both objectively measured and perceived balance. This is in agreement with previous studies noted that balance training has shown positive effects in healthy elderly people [Erreur ! Source du renvoi introuvable.Erreur ! Aucun nom n'a été donné au signet.].

A three-week multisensory training program was developing which was perceived would have a significant effect on postural sway control. This experimental study used a pretest-posttest control-group design. The study described a randomized controlled trial to test the hypotheses that a three-week multisensory training program had significant effects for postural sway control. The magnitude of differences of elderly training group (ETG) and elderly control group (ECG) for all three dependent variables of: (a) overall sway (OS), (b) medial-lateral sway (MLS), and (c) anterior-posterior sway (APS) after training were evaluated. Thus, the purpose of this study was to determine if the ETG improved in their postural sway measures when compared with the ECG. The researchers hypothesized that the ETG would show significant differences on postural sway measures (reduced sway) after completing the intervention when compared with the ECG.

The Chattecx Dynamic Balance System (CDBS) was used for the evaluation process and training program. In addition, the Berg Balance Test (BBT) was utilized to assess the functional balance skill before and after the training program for both groups. A second purpose of this study was to investigate if the improvement found on postural sway measures of ETG shown by the CDBS would indicate the same on the BBT scores when compared with ECG. The researchers hypothesized that the positive improvement of ETG produced by the CDBS could indicate similar training effect on the BBT.

\section{METHODS}

\subsection{Subjects}


A total of 24 non-injured elderly females were recruited. Volunteers were derived from local communities through posted advertisements. Subjects ranging from 60 to 80 years were selected from a homogeneous group that had body mass index (BMI) less than 30 in order to eliminate individuals with obesity. Inclusion criteria were normal ankles with no known injuries, normal visual and vestibular functions, normal musculoskeletal functions of all joints in the lower extremity, and free from all the neurological disease.

Prospective subjects were interviewed by telephone during the initial contact to identify their eligibility by answering a screening questionnaire. The first 24 qualified subjects were randomly assigned (drawing from an envelope) to the elderly training group (ETG) or the elderly control group (ECG). The randomization process helped confirmed that the treatment results would be less likely to be influenced by other external factors. A baseline assessment was conducted by completing a self-reported questionnaire of demographic variables, documenting health-history, types of sporting activities as well as hours spent on sporting activities per week to ensure that the ETG and the ECG were comparable and similar on all other factors especially for the ankle mechanics and posture.

The evaluation sessions (pretest and posttest) and the training sessions were arranged at subjects' convenience for both control and training groups. Following an explanation of the experimental procedures and a demonstration of the evaluation protocols and training conditions by the first author, subjects were required to read and sign an informed consent approved by ethics committee which approved all such investigations.

\subsection{Protocol}

Each training protocol was designed to enhance specific contributions to postural sway control. The process of sensorimotor rearrangement with subsequent postural stability was related to the degree of the initial instability. In addition, the literature has noted that the greater the initial risk of falling, the greater the percentage reduction in sway amplitudes with training [25]. Therefore, this three-week multisensory training program focused on manipulating or altered one or two of the three sensory systems (i.e. somatosensory input and visual input) to create postural balance instability. Somatosensory input was manipulated or altered by having each subject standing on a single leg or by keeping the platform moving. Visual input was eliminated by the eyes-closed (blindfolded) and was manipulated by 
watching a bull's-eye for visual feedback.

The nine training conditions were performed with the eyes-open and the eyes-closed; on a stable or a moving up or a moving down platform (40 tilt) with maximum speed ( 8.3 seconds per cycle); and, with bilateral and unilateral stance.

The primary author was the only trainer. Each condition was trained for one minute twice for the first set. The duration of one minute training for each condition was chosen because it was believed to be sufficient time for mechanoreceptors to respond with the stress of training. Subjects had a 30 seconds rest between repetitions. Approximately 30 minutes were required to complete all nine conditions with two repetitions at each session. Following a five-minute break, the set of nine conditions was repeated. The entire duration of training took approximately an hour for each session. The elderly training group was trained twice weekly three weeks. Subjects in both training and control groups were instructed to continue with their normal daily activities and not to initiate any new training programs or activities that could affect the results of this study for the duration of three week period after the pretest.

\subsection{Instrumentation and measurement}

\subsubsection{The Chattecx Dynamic Balance System (CDBS)}

The Chattecx Dynamic Balance System (CDBS) was used to objectively assess pre-training and post-training values for all subjects. In order to increase the first author's confidence in using the CDBS as an evaluation devise, the evaluation protocols were assessed in the previous study for test-retest reliability. The study finding indicated that the CDBS obtained good test-retest reliability (ICCs $>0.80$ ) on postural sway measures as clinical measures and the details have been published elsewhere.

\subsubsection{The Berg Balance Test (BBT)}

Both ECG and ETG were assessed using the Berg Balance Test (BBT) at pretest and posttest in order to determine whether the outcome measures produced by the laboratory measure (i.e. the CDBS) showing comparable results. This means that if the CDBS showed a decrease in all three postural sway measures in ETG after training, the BBT scores of these people would show an increase indicating that the ETG had better balance ability after the intervention when compared with the ECG. The BBT consists of 14 subtests performed in a standard order that assesses the ability to perform functional balance tasks [26]. Patients were asked to 
complete the 14 tasks, and each task was rated by primary author on a 5-point scale ranging from 0 (cannot perform) to 4 (normal performance). Overall scores could range from 0 (severely impaired balance) to 56 (excellent balance) [26]. Measurements obtained with this test have demonstrated excellent inter-rater and intra-rater reliability (i.e. ICCs 0.98 and 0.99, respectively) and a tendency toward at least moderately strong concurrent validity [27].

\subsection{Data analysis}

The average score of the two repetitions of each of the evaluation protocols were used to compute the total index of overall sway (OS), medial-lateral sway (MLS), and anterior-posterior sway (APS), for the pretest and posttest data. The means (M) and standard deviations (SD) of the pretest and posttest values for all three postural sway measures were calculated for both the control and training groups to compare the differences before and immediately after the three-week training program for the following training factors: (a) static balance with the eyes-closed condition; (b) dynamic balance with the eyes-open condition; (c) unilateral stance; (d) bilateral stance; (e) dominant leg, and (f) non-dominant leg. Independent $\mathrm{t}$-tests were performed on the pretest and posttest data. The $\mathrm{t}$ critical value ( $\mathrm{t}$ crit) of this study using two-tailed tests $(\alpha 2=0.05)$ and $22 \mathrm{df}(24-2)$ was $(\alpha 2=0.05)$ t $22= \pm 2.074$. For a t-ratio to represent a significant difference, the absolute value of the calculated ratio $(\mathrm{t}$ obs) must be greater than or equal to the critical value or $\mathrm{p} \leq 0.05$ to reject the null hypothesis. Standard Error of Measurement (SEM) and associated 95\% confidence intervals (95\% CI) were calculated to quantify measurement error or variation of individual scores. The significance level was set at $\mathrm{p} \leq 0.05$ a priori for all statistical tests unless otherwise specified.

\section{RESULTS}

\subsection{Training Effect for Types of Balance}

The t-statistic revealed significant differences between ECG and ETG for static balance with the eye-closed condition for OS, MLS, and APS ( $\mathrm{t}=7.765, \mathrm{p}=0.000 ; \mathrm{t}=7.264, \mathrm{p}=0.000 ; \mathrm{t}=8.464$, $\mathrm{p}=0.000$, respectively). Similarly, significant differences were found between ECG and ETG for dynamic balance with the eyes-open condition for OS, MLS, and APS ( $t=9.493, p=0.000$; $\mathrm{t}=7.376, \mathrm{p}=0.000 ; \mathrm{t}=9.731, \mathrm{p}=0.000$, respectively) after the three-week multisensory training intervention (Figure 1). 


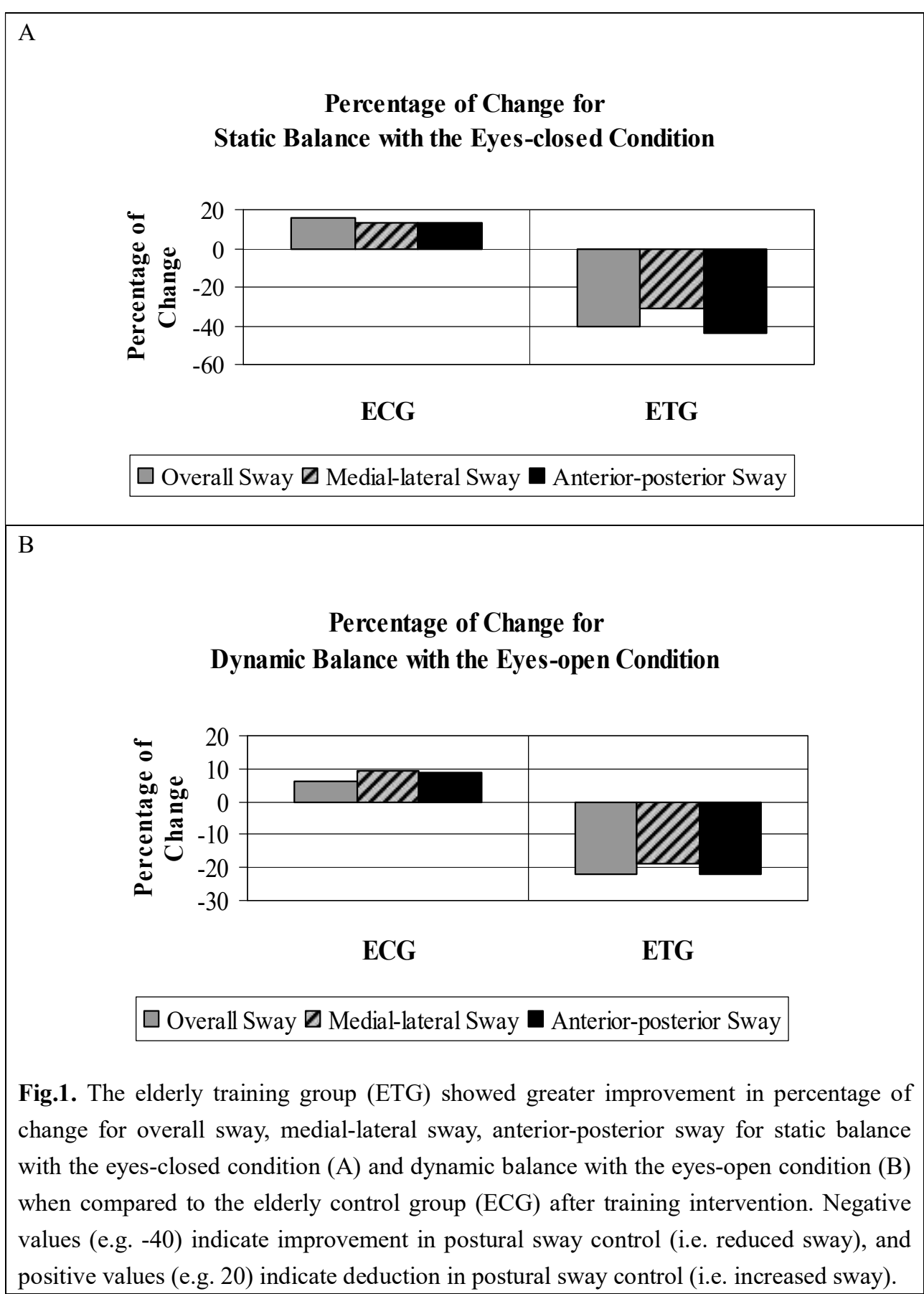

\subsection{Training Effect for Types of Stance}

The results revealed significant differences between ECG and ETG for OS, MLS, and APS for bilateral stance $(\mathrm{t}=8.894, \mathrm{p}=0.000 ; \mathrm{t}=6.207, \mathrm{p}=0.000 ; \mathrm{t}=8.548, \mathrm{p}=0.000$, respectively). For unilateral stance, the OS, MLS, and APS showed significantly differences $(\mathrm{t}=8.894$, $\mathrm{p}=0.000 ; \mathrm{t}=6.207, \mathrm{p}=0.000 ; \mathrm{t}=8.548, \mathrm{p}=0.000$, respectively) after the three-week multisensory 
training intervention (Figure 2).

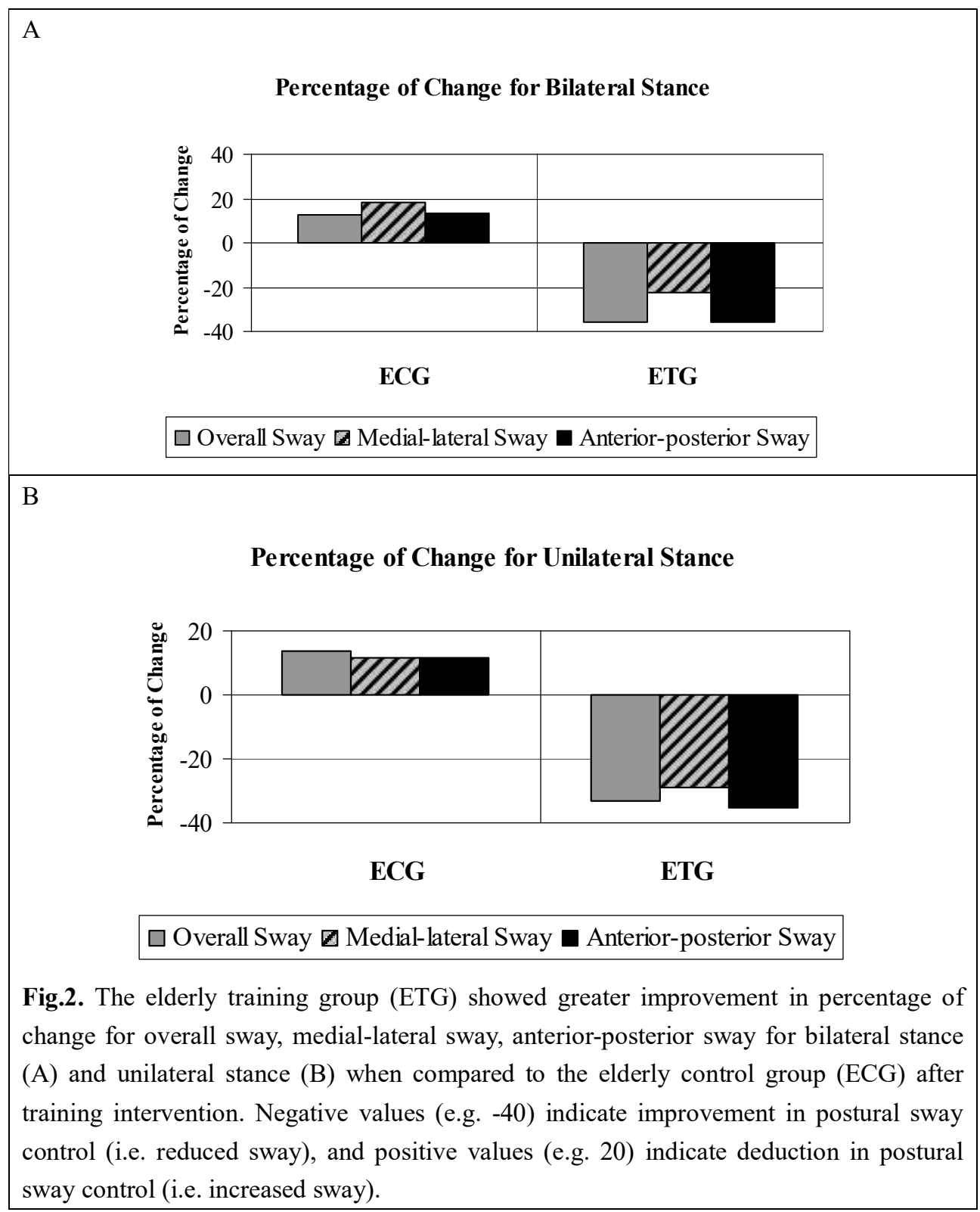

\subsection{Training Effect for Types of Leg Dominance}

The t-statistic revealed significant differences between ECG and ETG for OS, MLS, and APS at post-training for both the dominant leg $(\mathrm{t}=6.183, \mathrm{p}=0.000 ; \mathrm{t}=5.807, \mathrm{p}=0.000 ; \mathrm{t}=6.597$, $\mathrm{p}=0.000$, respectively) and the non-dominant $\operatorname{leg}(\mathrm{t}=7.672, \mathrm{p}=0.000 ; \mathrm{t}=8.441, \mathrm{p}=0.000$; $\mathrm{t}=7.229, \mathrm{p}=0.000$, respectively) (Figure 3 ). 


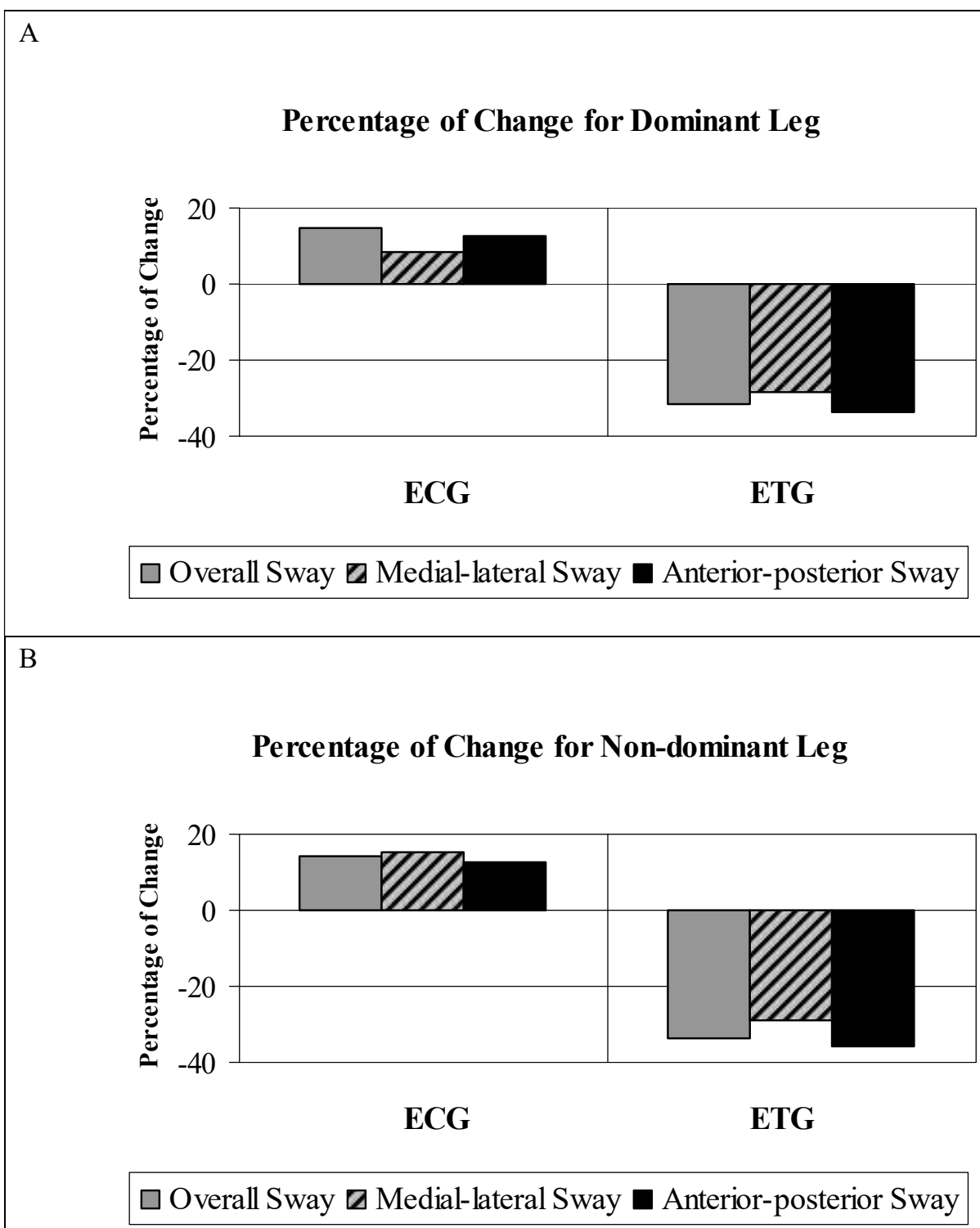

Fig.3. The elderly training group (ETG) showed greater improvement in percentage of change for overall sway, medial-lateral sway, anterior-posterior sway for dominant leg (A) and non-dominant leg (B) when compared to the elderly control group (ECG) after training intervention. Negative values (e.g. -40) indicate improvement in postural sway control (i.e. reduced sway), and positive values (e.g. 20) indicated deduction in postural sway control (i.e. increased sway).

\subsection{Training Effect on the Berg Balance Test (BBT)}

The mean difference in the total BBT scores between ETG and ECG (52.67 and 53, respectively) before training was not statistically significant at $\mathrm{t}(22)=0.364, \mathrm{p}=0.714$. After completing the three-week multisensory training intervention, the ETG revealed a significantly greater change in total BBT scores than the ECG (55.25 and 53, respectively), indicating a series of training conditions had led to increase total BBT scores at $\mathrm{t}(22)=$ 
$-4.075, \mathrm{p}=0.001$ (Figure 4).

The Berg Balance Test Score for

Pre-training and Post-training

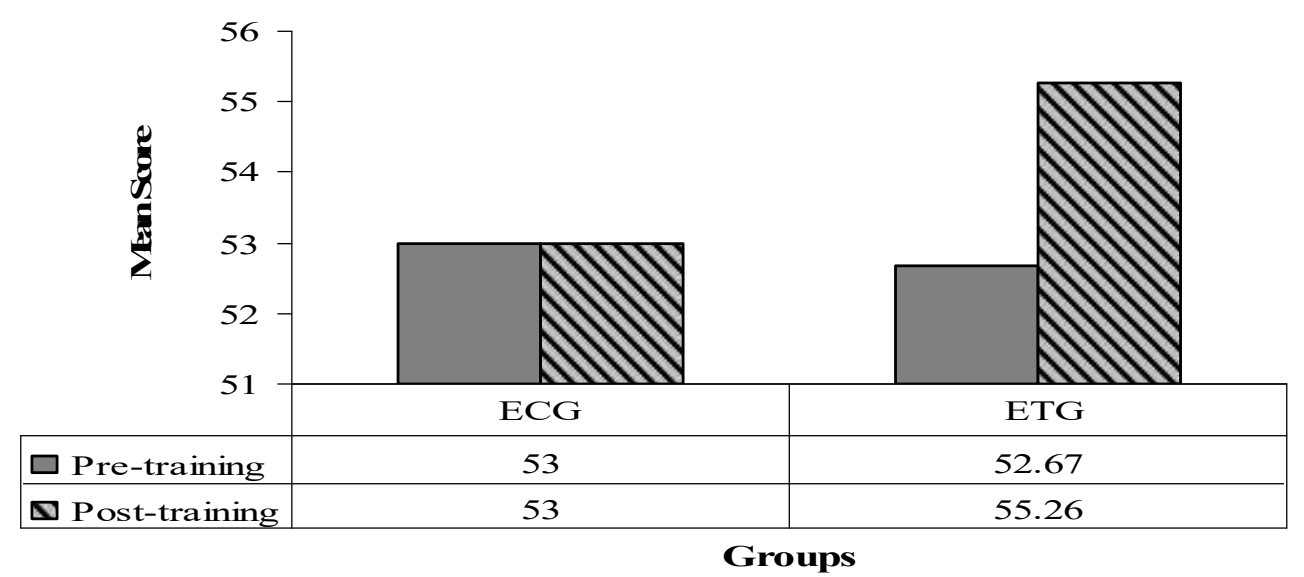

Fig.4. The Berg Balance Test score for pre-training and post-training of the three-week multisensory training intervention between the elderly control group (ECG) and the elderly training group (ETG).

\section{DISSCUSSION}

\subsection{Training effect on all six training factors}

Overall, there were no significant differences in pre-training values between ETG and ECG for their personal characteristics and postural sway. In addition, both the elderly groups' demonstrated similar pre-training results on BBT scores indicate they were equivalent for the measures recorded prior to the three-week multisensory training intervention.

This intervention trial demonstrated improvements in postural sway control in elderly non-injured females with exercise training. On average, the elderly training group improved (ranged $18.60 \%$ to $44.01 \%$ ) for all three outcome measures on all six training factors. For more details, see Figures 1, 2, and 3.

The positive training effects suggesting that the vestibular and somatosensory systems were able to fully compensate for the loss of visual inputs in the maintenance of balance and postural sway control. On the other hand, with the eyes-open condition, the visual inputs remained available but both vestibular and somatosensory inputs were minimized by setting the platform to various moving patterns and / or to a unilateral stance for the dynamic balance. The improved training effects showed that sensory information from the visual inputs could 
successfully compensate for the altered somatosensory and vestibular inputs. The possible mechanism for improved postural sway control is enhanced inter-sensory interactions and sensorimotor integration in the CNS. In the postural system model, balance skill training mainly affects the sensorimotor feedback pathway [4]. Since postural sway control significantly improved for the training conditions that required sensory interactions, processing improvement within the central integration mechanism is the underlying mechanism that is likely to have been responsible for balance improvement following training [Erreur ! Source du renvoi introuvable.Erreur ! Aucun nom n'a été donné au signet.]. The improvements were likely to be the result of the increased use of somatosensory, visual and vestibular information when performing the various training protocols under sensory deprivation conditions [21]. Another possible mechanism for this improvement is that the trained subjects were able to compare, select, and combine reliable sensory information for postural control more efficiently following the multisensory training [Erreur ! Source du renvoi introuvable.Erreur ! Aucun nom n'a été donné au signet.].

Previous studies have shown positive effects with balance training in healthy elderly adults [Erreur! Source du renvoi introuvable.Erreur! Aucun nom n'a été donné au signet.Erreur ! Aucun nom n'a été donné au signet.]. These studies supported the evidence that training programs with identified aims toward specific physiological systems related to postural control have consistently reported significant training effects. This is in agreement with the results of present study, suggesting that a training program designed to specifically improve sensory function was effective in the improvement of postural sway control and balance control of trained non-injured elderly females on six training factors of: (a) static balance with the eyes-closed condition, (b) dynamic balance with the eyes-open condition, (c) bilateral stance, (d) unilateral stance, (e) dominant leg, and (f) non-dominant leg.

\subsection{Training effect on the Berg Balance Test (BBT)}

The BBT is used as an indicator to predict risk of falling among elderly adults aged 60 years and above. According to the BBT indicator, the individual who scores below 45 has a higher risk of falling [26]. With respect of the BBT, it was found that the ETG had significantly higher scores than the ECG after the three-week multisensory training intervention. The ETG 
increased their total BBT scores from 52.56 at pre-training to 55 at post-training, whereas the pre-training and post-training of total BBT scores for the ECG remained unchanged. The average total BBT scores for the ETG were 55. Condron et al., [Erreur ! Source du renvoi introuvable.] suggested that measures of balance and postural sway on the CDBS, in particularly dynamic platform conditions using anterior-posterior tilting movement, were the most effective for accurate classification of fall risks in a sample with minimal to mild fall risk. The results of the present study suggest that the three-week multisensory training program was an effective means of improving balance ability and postural sway control, thus preventing the risk of falling and fall-associated injuries among elderly females. It is noteworthy to mention that the subjects in present study who had difficulty in BBT items such as Romberg stance, tandem stance, standing with eyes-closed gained greater improvement on the BBT scores at posttest after completing the training intervention.

The elderly females from the current study were free from any injuries or disorders of the CNS, reflected that they were healthy and non-injured elderly females. None of the subjects included received any specific training aimed at reducing postural sway or improving balance before the study started. This may explain the generally high motivation and good attendance in the ETG. Moreover, most of the subjects in ETG provided their best efforts when moving within their comfort range while training.

The improved postural sway control measured through the CDBS is consistent with the improved BBT shown by the ETG. It can be explained partly by the fact that both the CDBS and the BBT address the ability to hold a posture and maintain a position, as well as to change positions while keeping postural stability and balance. These improvements in postural sway control and balance ability along with enhanced BBT scores demonstrated the same treatment effects illustrated by the postural sway measures using the CDBS. The results of this study suggest that the training protocols used in the current study are an effective means of improving both the postural sway control (OS, and MLS, APS) and balance ability (BBT scores) for elderly females.

\section{CONCLUSION}

The positive results of the present study suggest that the three-week multisensory training 
program using the Chattecx Dynamic Balance System (CDBS) was able to successfully reduced postural sway, thus improved balance control ability. Balance exercises are essential to improve balance ability for all ages. Lee et al., (2016) [30] stated that balance exercises may improve balance ability of basketball players, thus reducing risk of injury. Therefore, this multisensory balance training program is in agreement that may be suitable to adapt as a fall prevention strategy program for elderly who present with a substantial risk of falling. Such a program would ensure significant lower incidences of falls. In turn, this would lead to substantially lower health related costs to falls and fall related injuries. Furthermore, the CDBS may be a key measure in early identification of fall risk for elderly adults. The information on postural sway improvement obtained from a laboratory measure (i.e. the CDBS) after training could be transferred to a field measure (i.e. the BBT scores) and indicates relevant finding from both measurement tools. Therefore, the BBT can be used to evaluate postural sway control as well.

\section{ACKNOWLEDGEMENTS}

This study was supported by Canadian Commonwealth World Fellowship Program and Academic Training Awards by Ministry of Education, Government of Malaysia to A.C. Lee.

\section{REFERENCES}

[1] Nashner L. Evaluation of postural stability, movement, and control. In: Hasson S, Ed. Clinical Exercise Physiology. Philadelphia: CV Mosby, 1994.

[2] Horak F B. Clinical measurement of postural control in adults. Phys Ther, 1987, 27(12), $1881-1885$.

[3] Irrgang J J, Whitney S L, Cox E D. Balance and proprioceptive training for rehabilitation of the lower extremity. Journal of Sport Rehabilitation, 1994, 3, 68-83.

[4] Kollmitzer J, Ebenbichler G R, Sabo A, Kerschan K, Bochdansky T. Effects of back extensor strength training versus balance training on postural control. Med Sci Sports Exerc, 2000, (10), 1770-1776.

[5] Dietz V. Role of peripheral afferents and spinal reflexes in normal and impaired human locomotion. Review Neurology (Paris), 1987, 143, 241-254. 
[6] Nardone A, Schieppati M N. Postural adjustment associated with voluntary contraction of leg muscles in standing man. Exp Brain Res, 1988, 69, 469-480.

[7] Mattacola C G, Lebsack D A, Perrin D H. Intertester reliability of assessing postural sway using the Chattecx Balance System. Journal of Athletic Training, 1995, 30 (3), 237-242.

[8] Horak F, Nashner L, Diener H. Postural strategies associated with somatosensory and vestibular loss. Program of Brain Research, 1990, 82, 167-177.

[9] Shepard N, Telian S. Practical management of the balance disorder patient. San Diego, London: Singular Publishing Group, Inc., 1996.

[10] Tinetti M, Speechley M. Prevention of falls among the elderly. N Engl J Med, 1989, 320, 1055-1059.

[11] $\mathrm{Hu} \mathrm{M}$, Woollacott M. Multisensory training of standing balance in older adults: II. kinematic and electromyographic postural responses. J Gerontol, 1994, 49(2), M62-M71.

[12] Kunesch E. Altered force release control in Parkinson's disease. Behavioral Brain Research 1995, 67, 43.

[13] Alexander N. Postural control in older adults. Journal of American Gerontology Social 1994, 2, 93-108.

[14] Frontera W, Meredith C, O'Reilly K. Strength conditioning in older men: skeletal muscle hypertrophy and improved function. J Appl Physiol, 1988, 64, 1038-44.

[15] Leenders N. The elderly. In: Ehrman J, Gordon P, Visich P, Keteyian S. Clinical Exercise Physiology. Champaign, IL : Human Kinetics, 2003, 571-587.

[16] Sheldon J. Effect of age on control of sway. Gerontology Clinic, 1963, 5, 129-138.

[17] Overstall P, Exton-Smith A, Imms Fa J A. Falls in the elderly related to postural imbalance. British Medicine Journal, 1977, 1, 261-264.

[18] Fernie G, Gryfe C, Holliday P, LLewellyn A. The relationship of postural sway in standing to the incidence of falls in geriatric subjects. Age and Aging, 1982, 11, 11-16.

[19] Fernie G, Holiday P. Postural sway in amputees and normal subjects. J Bone Joint Surg Am, 1978, 60A, 895-898.

[20] Woollacott M, Shumway-cook A, Nashner L. Postural reflexes and aging. In: Mortimer J, Editor. The aging motor system. New York: Praeger, 1982.

[21] $\mathrm{Hu} \mathrm{M}$, Woollacott M. Multisensory training of standing balance in older adults: I. 
postural stability and one-leg stance balance. J Gerontol, 1994, 49(2), M52-M61.

[22] Kammerlind A S C, Hakansson J K, Skogsberg M C. Effects of balance training in elderly people with non-peripheral vertigo and unsteadiness. Clin Rehabil, 2001, 15, 463-470.

[23] Ledin T, Kronhed A C, Moller C, Moller M, Odkvist L M, Olsson B. Effect of balance training in elderly people evaluated by clinical tests and dynamic posturography. J Vestib Res, 1991, 1, 129-131.

[24] Judge J, Lindsey C, Underwood M, Winsemius D. Balance improvment in older women: effects of exercise training. Phys Ther, 1993, 73(4), 254-265.

[25] Brandt T, Buchele W, Krafczyk S. Training effects on experimental posture instability: a model for clinical ataxia therapy. In: Bles W, Brandt T, Eds. Disorders of posture and gait. Amsterdam: Elsevier Science Publishers, 1986, 353-65.

[26] Berg K, Wood-Dauphinee S, Williams J, Gayton D. Measuring balance in the elderly: preliminary development of an instrument. Physiother Can, 1989, 41(6), 304-311.

[27] Berg K, Wood-Dauphinee S, Williams J. Measuring balance in the elderly: validation of an instrument. Canadian Journal of Public Health, 1992, Supplement 2, S2-S11.

[28] Bayouk J, Boucher J, Leroux A. Balance training following stroke: effects of task-oriented exercises with and without altered sensory input. Int J Rehabil Res 2006, 29, $51-9$

[29] Condron J, Hill K. Reliability and validity of a dual-task force platform assessment of balance performance: effect of age, balance impairment, and cognitive task. Journal of American Geriatrics Society, 2002, 50, 157-162.

[30] Lee AC, Kuang PF. The effectiveness of sports specific balance training program in reducing risk of ankle sprain in basketball. International Journal of Physiotherapy, 2016, $3(6), 681-686$.

\section{How to cite this article:}

Lee A. C. and Magee D. J. Multisensory Training for Postural Sway Control in Non-injured Elderly Females. J. Fundam. Appl. Sci., 2017, 9(6S), 1116-1131. 